\title{
Preferencias de los receptores de cuidados profesionales de larga duración sobre el sexo de la persona cuidadora ${ }^{1}$
}

Tomasa Báñez Tello²

Recibido: 12/06/2017 / Revisado: 12/06/2017 /Aceptado: 10/10/2017

Disponible on line

Resumen. En este artículo se analiza la importancia que el sexo de la persona cuidadora tiene para los receptores de cuidados profesionales de larga duración. Mediante la realización de una investigación cualitativa hemos recogido y analizado los testimonios de personas, cuidadas de forma profesional por hombres, para identificar sus preferencias sobre el sexo de la persona cuidadora y el papel que en esas preferencias puedan tener los discursos sociales dominantes sobre el cuidado, el género y la sexualidad. Tener en cuenta las opiniones y preferencias de las personas cuidadas es fundamental para una atención individualizada y el respeto de su intimidad.

Palabras clave: Personas cuidadas; cuidado profesional; hombres cuidadores

\section{[en] Preferences of recipients of long-term professional care with regard to the sex of carers}

\begin{abstract}
This article analyses the importance of the sex of carers to the recipients of long-term professional care. We have conducted qualitative research to compile and analyse the testimonies of individuals receiving professional care from men in order to identify their preferences as to the sex of the carer and the role that dominant social discourses regarding care, gender and sexuality may play in those preferences. Taking into account the opinions and preferences of care recipients is essential in providing individualised care and respecting the privacy of care recipients.
\end{abstract}

Key words: Care recipients; professional care; male carers.

Sumario: Introducción. 1. Metodología. 2. Resultados y discusión. 2.1. Persistencia de las construcciones sociales de género, sexualidad y cuidado. 2.2 El sexo del cuidador para a un segundo plano en el cuidado profesional. 3. Conclusiones. 4. Referencias bibliográficas.

Cómo citar: Báñez Tello, T. (2018).Preferencias de los receptores de cuidados profesionales de larga duración sobre el sexo de la persona cuidadora, en Cuad. trab. soc. 32(1), 49-60.

\section{Introducción}

Este artículo analiza la importancia que el sexo de la persona cuidadora tiene para los receptores de cuidados profesionales de larga duración. Una temática pertinente por la necesidad de afrontar la actual crisis de los cuidados con criterios de justicia social (Williams, 2012). Mediante una organización social de los cui- dados que los considere como responsabilidad pública, con la participación de las familias, la Administración Pública, las empresas y las entidades de la iniciativa social; que implique y comprometa a los hombres y que tenga en cuenta a las personas receptoras del cuidado, como sujetos activos, con capacidad de agencia y control de los procesos de cuidado.

1 Este artículo se ha elaborado en el marco de un proyecto de investigación financiado por RecerCaixa, un programa impulsado por la Obra Social 'la Caixa' con la colaboración de la Asociación Catalana de Universidades Públicas (2014ACUP00045).

2 Universitat de Barcelona, España

tomasabanez@ube.du 
El cuidado de larga duración es objeto de una creciente preocupación y debate por parte de investigadores, profesionales y gestores de la administración, de empresas y de entidades sociales, dada la crisis de los cuidados (Pérez-Orozco, 2006; Mandell, 2010; Benería, 2011). Esta crisis consecuencia del importante incremento de las necesidades de cuidado de larga duración provocado por los cambios socio-demográficos que han tenido lugar en los últimos años en Europa (Lethbridge, 2011; Abellán, Esparza y Pérez, 2011), y de la pérdida de capacidad para cuidar de las familias y de los entornos comunitarios y sociales, ha puesto en evidencia la falta de sostenibilidad y de equidad de la actual organización de los cuidados. Ante esta situación, se ha producido la externalización de los cuidados para su realización de forma remunerada por profesionales y organizaciones de diferentes sectores de la actividad económica. Pero manteniéndose la escasa visibilidad y valoración social de los trabajos de cuidado, con una presencia mayoritaria de mujeres y unas condiciones laborales precarias, como consecuencia del imaginario social que vincula el cuidado con los saberes femeninos (Torns, 2008; Tobío, Agulló, Gómez y Martín, 2010; Carrasco, Borderías y Torns, 2011; Torns, Borràs, Moreno y Recio, 2014).

El Trabajo Social como disciplina y como profesión tiene mucho que aportar a los actuales debates sobre la crisis de los cuidados y la necesidad de una organización social de los cuidados con criterios de justicia social. La perspectiva psicosocial y la proximidad y acompañamiento a las familias y personas, propias de nuestra profesión, nos proporciona un profundo conocimiento de las necesidades y situaciones de cuidado, pero también de las vivencias subjetivas de sus protagonistas y de los efectos de la implementación de las políticas públicas en la mejora de las condiciones y de la calidad de vida de las personas con necesidades de cuidado y en el mantenimiento y reproducción de situaciones de desigualdad.

Reflejo de este conocimiento, son numerosas las aportaciones realizadas desde el Trabajo Social tanto al análisis como a la mejora de los cuidados a personas en situación de dependencia. Se han analizado los cuidados familiares desde la perspectiva de género, poniendo en evidencia su falta de visibilidad (Julve, 2006) que contrasta con su importancia para la vida y la autonomía de las personas (Canimas,
2008; Rodríguez, 2013). En relación con los cuidados familiares, se han realizado y evaluado intervenciones de apoyo a los cuidadores familiares, con el objetivo de mejorar su calidad de vida y la atención a la persona cuidada (Fuentes y Moro, 2014). También se han analizado y realizado propuestas de mejora de los procedimientos de valoración de situaciones de dependencia (Oria, 2009; Erice y Arraztoa, 2010) y de los Planes Individualizados de Atención a la Dependencia (Caballero, 2010), con iniciativas como la gestión de casos (Rimbau, 2014). Por otra parte, son diversos los trabajos que analizan el papel de los servicios sociales en la atención a la dependencia y en la promoción de la autonomía de las personas en situación de dependencia (Romero, 2012) y que reivindican un sistema público de atención a la dependencia (Colom, 2006); Martín-Serrano, 2009; Pi Giménez, 2012).

Dada la temática de este artículo, queremos destacar las aportaciones realizadas desde el Trabajo Social en relación con la incorporación de las opiniones y vivencias de las personas receptoras de cuidados, con trabajos sobre las preferencias y capacidad de control que tienen las personas receptoras de cuidados profesionales realizados por hombres sobre su situación y sobre los cuidados (Báñez, 2017) o con el análisis de los puntos de vista de las personas mayores, con el objetivo de contribuir al cambio de la imagen negativa de la vejez y de visibilizar la dimensión emocional del cuidado (Arroyo y Soto, 2013). En relación con esta imagen negativa de las personas con necesidades de cuidado y de sus sentimientos ante las mismas, es interesante el análisis de como el cuidado, si se realiza respetando la dignidad de la persona, contribuye a su autonomía, en lugar de hacerla dependiente, ya que frente al ideal de independencia predominante en los discursos sociales dominantes sobre el cuidado, es la interdependencia y la autonomía relacional la que nos caracteriza como seres humanos (Cordero y Palacios, 2017).

\section{Organización social del cuidado con crite- rios de justicia social}

El concepto de social care propuesto por Mary Daly y Jane Lewis implica la consideración del cuidado como responsabilidad pública, en contraste con las conceptualizaciones que lo circunscribían al ámbito familiar, enfatizando la dimensión afectiva y moral y naturalizán- 
dolo como responsabilidad de las mujeres. De acuerdo con la propuesta de estas autoras, el cuidado social es:

El conjunto de las actividades y las relaciones que intervienen en la satisfacción de las necesidades físicas y emocionales de las personas adultas dependientes y de los niños, y los marcos normativos, económicos y sociales en los que aquellas se asignan y se desarrollan (2000, p. 284).

Señalan además su naturaleza compleja, ya que el cuidado definido de esta forma, incluye tres dimensiones diferentes: es una forma de trabajo, tiene un componente de responsabilidad y de obligación y supone unos costes económicos y afectivos. A partir de esta propuesta conceptual se ha extendido el uso del término trabajos de cuidado, para referirse al trabajo de cuidado no remunerado realizado por familiares y al trabajo profesional remunerado en la administración pública, en empresas y en entidades de la iniciativa social (Himmelweit, 1995).

El diseño y la implementación de una organización social de los cuidados, que responda a criterios de justicia social, requieren en primer lugar, la profesionalización de este sector, con condiciones laborales y salarios dignos. Para ello, es necesario cambiar el imaginario social que asocia el cuidado con el trabajo doméstico y familiar y construir una estructura profesional de los trabajos de cuidados (Recio, Moreno, Borràs y Torns, 2015). La implementación de estas propuestas requiere gestionar la complejidad y el carácter relacional del cuidado, a la hora de definir las actividades que forman parte del trabajo de cuidado (Cameron y Moss, 2007; Anxo y Fagan, 2005; Prieto y Serrano, 2013); así como determinar las competencias técnicas, habilidades de relación, destrezas afectivas y orientaciones éticas que se requieren para cuidar de forma profesional y remunerada (Tobío, Agulló, Gómez y Martín, 2010), diferenciándolas de las cualidades que los estereotipos de género atribuyen a las mujeres y superar la aparente contradicción que supone el reconocimiento formal de la formación, con el hecho de que el cuidado implica además cualidades personales (Zelizer, 2009; Recio, Moreno, Borràs y Torns, 2015).

Otro de los elementos clave para la implementación de una organización de los cuidados que responda a criterios de justicia social y que permita afrontar la crisis de los cuidados, es la implicación de los hombres en el cuidado de larga duración tanto familiar, como remunerado. Los resultados de la investigación dirigida por Dolors Comas D'Argemir en Cataluña (2015-2017) sobre hombres cuidadores muestran un incremento de la presencia de hombres en el cuidado familiar de larga duración, como consecuencia de la crisis de los cuidados y de la crisis económica; así como de la reactivación del papel protector del parentesco, quedando en un segundo plano los roles de género. Las precarias condiciones del mercado laboral, el desempleo o la jubilación son factores que facilitan la conversión de los hombres en cuidadores principales familiares (Soronellas y Comas-D'Argemir, 2017).

Según estos mismos resultados, la profesionalización de los trabajos de cuidados y el impacto de la crisis económica en el empleo, han supuesto la incorporación de los hombres a los trabajos remunerados de cuidado. Se trata de hombres que, como consecuencia de la crisis económica, han reconducido sus trayectorias laborales desde ocupaciones fuertemente masculinizadas hacia los trabajos remunerados de cuidado y que al hablar de su trabajo como cuidadores remunerados enfatizan el distanciamiento emocional, la fuerza física y la dimensión técnica de sus tareas como estrategia de afirmación de su identidad masculina. En este sentido insisten en que el hecho de ser hombres no es un factor que influya en como realizan su trabajo, un trabajo que sí que está condicionado por factores como el sexo y las necesidades de cuidados de la persona receptora, el ámbito, el tipo de recurso y el espacio en el que se realiza el cuidado. En contraste con esta visión de su trabajo, los resultados de esta investigación señalan que esta creciente presencia de los hombres en los cuidados remunerados reproduce las relaciones de género, ya que en sus discursos enfatizan la fuerza física y las dimensiones técnicas del cuidado; así como las resistencias de las mujeres a ser cuidadas por un hombre (Bodoque, Roca y Comas-d'Argemir, 2016, p. 85), especialmente cuanto el cuidado se realiza en el domicilio, por respeto a la privacidad e intimidad (Roca y Bodoque, 2017, p. 14).

Finalmente, el diseño e implementación de una organización social de los cuidados que responda a criterios de justicia social y que permita gestionar de forma adecuada la complejidad y diversidad del cuidado requie- 
re tener en cuenta las opiniones y preferencias de las personas receptoras en relación con el cuidado, en particular con la higiene personal, dada la importancia del respeto a la intimidad, en especial la corporal y física, en el cuidado de las personas en situación dependencia (Tobío, Agulló, Gómez y Martín, 2010; Carrasquer, 2013). Dado que la vivencia del pudor es subjetiva y no depende solo de la exposición corporal y del tipo de actividad cuidado, para que los cuidadores accedan a la vida privada de las personas cuidadas es preciso contar con su consentimiento y tener un profundo conocimiento de la persona, de su manera de vivir la intimidad y de sus temores, para que el cuidado no sea vivido como una amenaza a su intimidad (Martínez, Díaz y Sánchez, 2016).

\section{Metodología}

Los resultados que analizamos en este artículo forman parte de una investigación interdisciplinar (Antropología Social, Trabajo Social, Comunicación) más amplia, realizada en el período 2015-2017 en Cataluña. Con el objetivo de identificar las barreras culturales y de oportunidad que obstaculizan la implicación de los hombres tanto en el trabajo de cuidado no remunerado cómo en el remunerado, así como de detectar los modelos emergentes de cambio en las relaciones de género tanto en el ámbito laboral, como doméstico y comunitario. Se trata de un estudio en el que además de enfatizar la importancia de la implicación de los hombres en los trabajos de cuidados, hemos tenido en cuenta a las personas receptoras de cuidado, como sujetos activos, con capacidad de agencia y control de los procesos de cuidado y cuyos derechos se encuentran a menudo en tensión con los de otros actores como por ejemplo las personas cuidadoras (Comas D'Argemir, 2016).
Aunque en este artículo presentamos únicamente el análisis de las preferencias de las personas receptoras de cuidados profesionales de larga duración en relación al sexo de la persona cuidadora, hemos publicado con anterioridad diversos trabajos, tanto de reflexión teórica sobre la temática de estudio (Comas, 2014; 2015), como sobre los resultados de la investigación en cuanto a la presencia de hombres en los cuidados familiares (Soronellas y Comas-d'Argemir, 2017) y en los cuidados profesionales (Bodoque, Roca y Comas-d'Argemir, 2016), a los puntos de vista de los gestores y responsables de políticas públicas (Roca y Bodoque, 2017) y en relación con las preferencias y capacidad de control que tienen las personas receptoras de cuidados sobre su situación y sobre los cuidados (Báñez, 2017).

Hemos realizado el análisis de las preferencias de las personas receptoras de cuidados profesionales de larga duración ${ }^{3}$ en relación al sexo de la persona cuidadora a partir de los testimonios recogidos mediante la realización de entrevistas en profundidad, con el objetivo de conocer las opiniones y vivencias de las personas receptoras de cuidados profesionales de larga duración sobre el cuidado y en especial, dado el enfoque de la investigación, sobre sus preferencias en cuanto al sexo de la persona cuidadora. Todas las entrevistas fueron grabadas y transcriptas posteriormente. Además, de forma previa a la realización de la entrevista, las personas participantes fueron informadas de los objetivos del estudio y firmaron un consentimiento informado en el que dejaban constancia de su voluntariedad y libertad de respuesta. Todas las personas a las que solicitamos participar en la investigación accedieron a ser entrevistadas.

La guía de la entrevista diseñada específicamente para este estudio, incluía los siguientes aspectos: datos de identificación de la persona entrevistada (edad, lugar de nacimiento y de residencia, tipo de domicilio, estado civil,

En esta investigación asumimos la definición cuidados de larga duración realizada por la Organización Mundial de la Salud (2000): "Los cuidados de larga duración son el sistema de actividades llevadas a cabo por cuidadores informales (familiares, amigos o vecinos) o profesionales (sanitarios, sociales u otros), o ambos, para conseguir que una persona que no sea totalmente capaz de cuidar de sí misma mantenga la mejor calidad de vida posible, de acuerdo con sus preferencias individuales, con el mayor grado posible de independencia, autonomía, participación, realización personal, y dignidad humana.” (8)

En el caso de España los cuidados de larga duración se concretan en el denominado sistema de atención a la dependencia, diseñado por la Ley 39/2006, de 14 de diciembre, de Promoción de la Autonomía Personal y Atención a las personas en situación de dependencia. Una normativa que define la dependencia, de acuerdo con la propuesta del Consejo de Europa, como "El estado de carácter permanente en que se encuentran las personas que, por razones derivadas de la edad, la enfermedad o la discapacidad, y ligadas a la falta o a la pérdida de autonomía física, mental, intelectual o sensorial, precisan de la atención de otra u otras personas o ayudas importantes para realizar actividades básicas de la vida diaria o, en el caso de las personas con discapacidad intelectual o enfermedad mental, de otros apoyos para su autonomía personal". 
composición familiar, estudios, ocupación, valoración subjetiva de su clase social, creencias, motivos por los que necesita el cuidado y relación con la persona cuidadora); vivencias de la persona cuidada (la necesidad de cuidados, su identidad como persona, sus experiencias de cuidar y de autocuidado, su capacidad de decidir sobre el cuidado que necesita y que recibe); la persona cuidadora (que tareas de cuidado realiza, en caso de que sea un cuidador remunerado, quién y como lo ha contratado, en caso de que sea un cuidador no remunerado, qué apoyos recibe, características de la persona cuidadora (sexo, edad, nacionalidad, etc.), preferencias respecto a la persona cuidadora; relación con la persona cuidadora y expectativas y satisfacción respecto a la persona cuidadora; los cuidados que recibe (que entienden por cuidado, cuáles son sus necesidades de cuidado, cuidados que recibe, capacidad de negociar y controlar estos cuidados).

Las tareas de contacto con las personas receptoras de cuidados se realizaron a través de las entidades colaboradoras en la investigación ${ }^{4}$ que también han participado de forma activa en los talleres de debate de los resultados y conclusiones del estudio y de diseño de las estrategias y actuaciones para la transformación y mejora de la organización social de los trabajos de cuidados desde una perspectiva de justicia social.

El análisis que hemos realizado de las preferencias de los receptores de cuidados, en cuanto al sexo de la persona cuidadora, ha estado orientado por las siguientes preguntas ¿le importa a la persona cuidada el sexo del cuidador? y esta importancia $i$ depende del sexo de la persona cuidada?, y ha sido realizado a partir de unas categorías analíticas basadas en sus testimonios y elaboradas a partir del razonamiento inductivo y con el apoyo del programa informático Atlas.ti 7. Estas categorías analíticas se refieren a lo que las personas cuidadas entienden por cuidado (higiene personal, ayuda en las actividades de la vida diaria, escucha y atención, apoyo emocional), a lo que valoran respecto al cuidado que reciben (cualidades personales, trato, profesionalidad, eficacia) y sus valoraciones de las experien- cias de cuidado profesional. Por otra parte, en el análisis de los testimonios recogidos hemos tenido en cuenta como variables el sexo y edad de la persona cuidada, los motivos del cuidado, el tipo de cuidador y lugar donde se reciben los cuidados.

Las 43 personas entrevistadas son receptores de cuidados profesionales de larga duración realizados por hombres y residentes en Barcelona y su área metropolitana. De estas 43 personas, 37 han manifestado sus opiniones sobre la importancia para el cuidado del sexo de la persona cuidadora. Se trata de hombres (22) y mujeres (15); cuidados en una residencia (5), en una unidad hospitalaria de agudos de salud mental (24) y en sus domicilios (8); por cuidadores remunerados (31), por asistentes personales ${ }^{5}$ (3) y por voluntarios (1); los motivos del cuidado son un diagnóstico de salud mental (26), personas mayores de 65 años con necesidades de apoyo para su desempeño en la vida diaria (8) y diversidad funcional (5).

\section{Resultados y discusión}

De las 37 personas receptoras de cuidados que hablan de sus preferencias en relación al género de la persona cuidadora, 19 personas, no manifiestan ninguna preferencia; 14 personas manifiestan que prefieren que les cuide una mujer y 4 personas prefieren que les cuide un hombre. De las 19 personas que manifiestan que les es indiferente el sexo de la persona cuidadora, 13 son hombres y 6 mujeres. 13 de estas personas tienen un diagnóstico de salud mental, 3 son personas con diversidad funcional y 3 son personas mayores con necesidades de apoyo para su desempeño en la vida diaria. 12 personas son atendidas en una unidad hospitalaria de agudos de salud mental, 2 personas son atendidas en una residencia y 5 personas son atendidas en sus domicilios, de las cuales dos también reciben atención en un centro de día. 14 personas con atendidas por cuidadores remunerados, 3 por asistentes personales y una persona es cuidada por su hijo. De las 14 personas que prefieren ser cuidadas por mujeres, 5 son hombres y 9 mujeres. 10 de estas per-

Fundación Catalunya La Pedrera (Programa REMS: Reforzar y Estimular la Memoria y la Salud), Corporación Sanitaria Parc Taulí, Federación Salud Mental Catalunya, Oficina de Vida Independiente, ECOM (Federación de organizaciones de persones con discapacidad física) y Residencia de personas mayores Betania.

Aunque los asistentes personales también son profesionales remunerados, se ha especificado dadas las implicaciones que para la capacidad de agencia de la persona receptora del cuidado tiene el ser ayudada por un cuidador o por un asistente personal, de acuerdo con la perspectiva de vida independiente. 
sonas tienen un diagnóstico de salud mental y 3 son personas mayores con necesidades de apoyo para su desempeño en la vida diaria. 9 personas son atendidas en una unidad hospitalaria de agudos de salud mental, 3 personas son atendidas en una residencia y una persona es atendida en su domicilio. 12 personas son atendidas por cuidadores remunerados y una persona es cuidada por su hijo. Las cuatro personas que prefieren ser cuidadas por un hombre son todos hombres, dos de ellos con un diagnóstico de salud mental y las otras dos son personas mayores con necesidades de apoyo para su desempeño en la vida diaria. Las dos personas con diagnóstico de salud mental son cuidadas en una unidad hospitalaria de agudos de salud mental por cuidadores remunerados. Las dos personas mayores con necesidades de apoyo para su desempeño en la vida diaria son cuidadas en sus domicilios, en un caso por un cuidador remunerado y en el otro por una persona voluntaria.

\subsection{Persistencia de las construcciones so- ciales de género, sexualidad y cuidado}

Los discursos de las personas cuidadas de forma profesional por hombres que hemos analizado muestran la persistencia de las construcciones sociales de género, sexualidad y cuidado, que sitúa la responsabilidad del cuidado únicamente en el ámbito familiar, enfatizando sus dimensiones afectivas y morales y adjudicando las tareas de cuidado a las mujeres, en virtud de su naturaleza femenina (Torns, 2008; Tobío, Agulló, Gómez y Martín, 2010; Carrasco, Borderías y Torns, 2011).

De esta forma, se continúan asociando al cuidado cualidades naturalizadas como femeninas (prácticas, detallistas, comunicativas) en especial para las tareas de higiene personal y limpieza. Esto es lo que reflejan los testimonios de Magui de 87 años, atendida en una residencia, de María de 85 años, atendida en un centro de día y de Ricardo de 55 años, ingresado en una unidad hospitalaria de agudos de salud mental:

El trato lo noto diferente cuando me atiende un hombre o una mujer. Irene (es una de las cuidadoras de la residencia) siempre me abraza y me da besos mientras me atiende (Magui).

Las mujeres nos fijamos más en los detalles, en lo que hay que hacer, vemos las cosas .... Y el hombre en esto es más despistado (...) creo que es diferente como cuida un hombre a como cuida una mujer (María).

Como cuidadores, creo que la mano femenina es más delicada que la masculina. (...) es una cosa genética (...) las mujeres tratan con más suavidad que los hombres que somos más bruscos. A mí me gusta más que me trate una mujer que un hombre. (...) aunque aquí en el hospital, todos los profesionales están muy acreditados y bien preparados para hacer su trabajo (Ricardo).

Este último testimonio, en el que después de enfatizar las cualidades femeninas para el cuidado, se destaca la preparación que los cuidadores profesionales, hombres y mujeres, tienen para realizar su trabajo, parece que pone en evidencia la aparente contradicción que supone el reconocimiento formal de la formación, con el hecho de que el cuidado implica también cualidades personales, que los estereotipos de género atribuyen a las mujeres (Recio, Moreno, Borràs y Torns, 2015).

En los discursos de las personas cuidadas aparece también la multidimensionalidad del cuidado, con referencias a tareas instrumentales y a la dimensión relacional, destacándose cualidades feminizadas y masculinizadas dependiendo de las diferentes dimensiones. De esta manera se justifica la presencia de hombres en el cuidado con referencias a su fuerza física para la realización de las tareas que requieren esfuerzo y a la afinidad, cuando cuidan a hombres, en cuanto los temas de conversación como por ejemplo del futbol. Esto es lo que manifiesta Martin de 65 años, con una discapacidad física y atendido en su domicilio por un cuidador remunerado:

Yo para que me cuiden preciso una persona con una cierta fuerza física. (...) Otra ventaja de ser cuidado por un hombre es que podemos hablar de futbol (se ríe) Pero las mujeres son mejores para la limpieza, porque son mucho más prácticas. (Martín).

Testimonios como este ponen en evidencia la importancia de definir las actividades que forman parte de los trabajos de cuidado, teniendo en cuenta su carácter relacional (Cameron y Moss, 2007; Anxo y Fagan, 2005; Prieto y Serrano, 2013), para poder avanzar en la determinación de las competencias y habilidades que se requieren para cuidar de forma profesional y remunerada (Tobío, Agulló, Gómez y Martín, 2010), diferenciándolas de las cualida- 
des que los estereotipos de género atribuyen a las mujeres. Esta definición de las actividades que forman parte de los trabajos remunerados de cuidado contribuiría a superar la dificultad que para la profesionalización del cuidado supone su asimilación con las tareas domésticas y no con el saber experto (Recio, Moreno, Borràs y Torns, 2015). Una diferenciación que sí que realiza en el cuidado en contextos institucionales, pero no en el servicio de asistencia domiciliaria (Bodoque, Roca y Comas-d'Argemir, 2016).

Un importante número de mujeres cuidadas (9 de 15) manifiestan que prefieren ser cuidadas por mujeres, sobre todo en lo que se refiere a la higiene personal por una cuestión de pudor y vergüenza, aunque reconocen que la formación prepara por igual a hombres y mujeres para ser cuidadores y que han tenido experiencias positivas de haber sido cuidadas por un hombre. Esto es lo que expresan Daniella de 70 años, atendida en una unidad hospitalaria de agudos de salud mental y Antonia de 79 años, atendida en una residencia:

No he notado un trato diferente por parte de cuidadores hombre y mujeres (...) Pero prefiero que me cuide una mujer (...) Por pudor, por machismo... No sé... (Daniella).

Es que a mí no me hace gracia que me toquen los hombres, es por vergüenza (...) no es que crea que no lo van a hacer bien, (...) es su trabajo, $(. .$.$) han hecho sus estudios para hacer-$ lo y yo no tengo ninguna cosa que decir de eso. Tienen derecho a trabajar, lo mismo que las mujeres tienen derecho a hacer trabajos que antes hacían solo los hombres y ahora pues los hacen también las mujeres (Antonia).

Algunas de estas mujeres que prefieren ser cuidadas por una mujer, en especial en lo que se refiere a la higiene personal, expresan que para el resto de las tareas del cuidado el sexo de la persona cuidadora les es indiferente. Esto es lo que manifiestan Desirée de 44 años y Elisabeth de 42 años, ambas atendidas en una unidad hospitalaria de agudos de salud mental:

Me da exactamente lo mismo, porque lo puede hacer bien tanto un hombre como una mujer. (...) Pero no es lo mismo que te bañe un hombre a que te bañe una mujer. Por el pudor, porque soy un poco pudorosa para que me bañen (...), pero saltada esta barrera, si, da lo mismo. (Desirée).
Una mujer (...) para la higiene personal una mujer, para las otras tareas me sería indiferente. (Elisabeth).

Todas las personas que prefieren ser cuidadas por hombres (4) son hombres y lo justifican con referencias a la vergüenza o pudor y a la comodidad, en especial en lo que refiere a la higiene personal. Es lo que manifiesta Feliu de 85 años, atendido por un cuidador remunerado en su domicilio:

Yo prefiero un hombre, no precisamente porque me vaya a cuidar mejor, puede ser, al contrario, puede que lo haga peor, pero yo me siento más libre y cómodo con un hombre como cuidador que con una mujer (Feliu).

El análisis de estos testimonios muestra la persistencia de los estereotipos sobre la sexualidad en las preferencias para ser atendidos por una persona del mismo sexo, en especial para las tareas de higiene personal, aunque a la vez se exprese que ,para el resto de tareas de cuidado, les es indiferente ser atendidos por un hombre o por una mujer, dada su formación para hacer este trabajo de forma profesional. Poniendo de manifiesto la importancia de que para que los cuidadores accedan a la vida privada de las personas cuidadas es preciso conocer su manera de vivir la intimidad, sus temores y sus preferencias y contar con su consentimiento, para que el cuidado no sea vivido como una amenaza a la intimidad, ya que ya que la vivencia del pudor es subjetiva (Martínez, Díaz y Sánchez, 2016).

\subsection{El sexo del cuidador para a un segundo plano en el cuidado profesional}

Pero junto a esta persistencia de las construcciones sociales de género, sexualidad y cuidado, nuestros resultados muestran que un número importante de personas cuidadas, 19 de 37, manifiestan que les es indiferente el sexo de la persona cuidadora, ya que consideran que su preparación, eficiencia y profesionalidad son más importantes que el que sea hombre o mujer. Se trata de receptores de cuidado de larga duración, que mayoritariamente son cuidados por profesionales en una institución (unidad hospitalaria de agudos de salud mental, residencia o centro de día). En estos contextos institucionales el cuidado se realiza por parte de profesionales y las personas cui- 
dadas no tienen normalmente la posibilidad de elegir al cuidador, con lo que pueden tener la experiencia de ser cuidadas tanto por hombres como por mujeres. Los resultados que presentamos en este artículo, parecen indicar que, en el cuidado profesional, realizado en contextos institucionales, el sexo del cuidador queda en un segundo plano, enfatizándose en cambio la preparación, eficiencia y profesionalidad de los cuidadores. Es decir, la profesionalización de los trabajos de cuidado, en la medida en que supone la determinación de las competencias técnicas, habilidades de relación, destrezas afectivas y orientaciones éticas que se requieren para cuidar de forma profesional y remunerada (Tobío, Agulló, Gómez y Martín, 2010), diferencia estas competencias de las cualidades que los estereotipos de género atribuyen a las mujeres, facilitando de esta forma que el sexo de la persona cuidadora pase a un segundo plano. Esto es lo muestran los testimonios de Jonathan de 38 años y Gala de 35 años, ambos atendidos en una unidad hospitalaria de agudos de salud mental:

A mí me es indiferente que me cuide un hombre o una mujer, mientras sea buen profesional y sepa realmente lo que se hace (Jonathan).

Siempre que sean eficientes y profesionales, me es indiferente que me cuide un hombre o una mujer, lo que me importa es que sean personas eficientes (Gala).

Otras personas, que manifiestan no tener preferencias en relación al sexo de la persona cuidadora, señalan que lo que consideran importante, incluso cuando se trata de profesionales, no es el sexo sino el trato y las características personales. Entre las que destacan la empatía, que sea agradable, buena persona, atento, que tenga paciencia, que sea una persona equilibrada. Esto es lo que manifiestan Casimiro de 81 años y Dorian de 41 años, ambos atendidos en una unidad hospitalaria de agudos de salud mental:

Yo primaría el carácter de la persona, que sea buena persona (...) la personalidad es más importante que el hecho de ser hombre o mujer (Casimiro).

La paciencia y, bueno, el... como se dice, el equilibrio. Una persona equilibrada y con paciencia (Dorian).
Además de destacar estas cualidades personales como más importantes que el sexo de la persona cuidadora, las personas con diversidad funcional entrevistadas, señalan la importancia que para ellas tiene el poder sentirse a gusto, tener afinidad con el asistente personal y que éste respete sus decisiones en relación con el cuidado. Esto es lo que expresan Agustín de 42 años y Oscar de 38 años, ambos con discapacidad física y ayudados por asistentes personales:

A mí me es indiferente contratar (como asistente personal) a un hombre, mujer, persona transexual (...). El sexo no es la cuestión central. (...) Lo que valoro es sentirme a gusto con aquella persona, porque estará contigo en mis círculos de intimidad, (...) que haya afinidad, que es algo que por desgracia no se puede construir tan fácilmente (Agustí).

Para mí, más importante que el sexo del asistente personal, es que sobre todo no se meta en mis decisiones y, si me equivoco, me equivoco yo. No se equivoca el asistente personal. Respetar mi propia decisión (Oscar).

Estos testimonios ponen en evidencia la complejidad de los trabajos de cuidado y la importancia de la dimensión relacional, más allá de las tareas y contenidos instrumentales (Anxo y Fagan, 2005; Cameron y Moss, 2007; Prieto y Serrano, 2013); así como el reto de que la atención profesional en contextos institucionalizados no suponga la despersonalización del cuidado (Wellin y Jaffe, 2004) y la pérdida de la capacidad de agencia de las personas cuidadas (Recio, Moreno, Borràs y Torns, 2015); y la necesidad de superar la aparente contradicción del reconocimiento formal de la formación de los cuidadores, con el hecho de que el cuidado implica además cualidades personales (Zelizer, 2009,Recio, Moreno, Borràs y Torns, 2015).

Algunas de las personas entrevistadas señalan la importancia del lugar en el que se realiza el cuidado, manifestando que, para ser atendidos en una unidad hospitalaria de agudos de salud mental, el sexo de la persona cuidadora les es indiferente, pero que, para el cuidado en sus domicilios, prefieren una mujer, por pudor y vergüenza al tratarse de tareas y espacios relacionados con su intimidad. Esto es lo que manifiesta Estela de 53 años, atendida en la unidad hospitalaria de agudos de salud mental: 
En este caso, los profesionales que me trata aquí en el hospital me es igual que sean hombres o mujeres. Pero, para cuidarme en casa prefiero una mujer, ya que si viene un hombre me sentiría mal. Por vergüenza (Estela).

Estos testimonios son coherentes con los trabajos que señalan que las personas cuidadas de forma profesional a través de los servicios de atención domiciliaria prefieren ser atendidos por personas del mismo sexo, en especial las mujeres que muestran sus resistencias a la realización de higiene personal por parte de un cuidador (Roca y Bodoque, 2017).

Algunas de las personas cuidadas entrevistadas justifican que les es indiferente el sexo de la persona cuidadora haciendo referencia a sus experiencias personales satisfactorias de haber sido cuidados de forma profesional tanto por hombres como por mujeres. Esto es lo que expresan Mariano de 86 años, atendido en una residencia, Eugenia de 86 años y Dalia de 45 años, atendidas en una unidad hospitalaria de agudos de salud mental:

Estoy contento. Aquí hay unas empleadas muy buenas, y los empleados también, y todos son muy atentos (Mariano).

Todos (profesionales hombres y mujeres) me trataron muy bien (Eugenia).

Hombre, con las mujeres siempre parece que al ser mujer hay más cariño, hay otro tipo de trato, ¿no? (...) Pero en mi experiencia en el hospital yo me he encontrado con enfermeras estrictas y enfermeros muy simpáticos y dulces (Dalia).

Es interesante como, en este último testimonio, la persona cuidada contrasta lo que, de acuerdo con el estereotipo de género, se puede esperar de una mujer y de un hombre en cuanto al trato y la relación, con su experiencia personal de haber sido atendida por profesionales de ambos sexos. Dado que los discursos de las personas cuidadas cuyos análisis presentamos en este artículo, parecen indicar que, en el cuidado profesional, realizado en contextos institucionales el sexo del cuidador queda en un segundo plano, planteamos la cuestión de si el hecho de que estos contextos posibiliten la experiencia del cuidado por parte de un hombre, facilita el que las personas cuidadas prescindan de los estereotipos de género en relación al cuidado. Una cuestión que también se ha identificado en algunos trabajos de investigación sobre los hombres cuidadores remunerados en el servicio de asistencia domiciliaria, quienes manifiestan que las reticencias iniciales que la mayor parte de las mujeres cuidadas tienen de ser atendidas por un hombre, no solo desaparecen con el tiempo, sino que acaban valorando positivamente el cuidado recibido (Roca y Bodoque, 2017, p. 14).

\section{Conclusiones}

El análisis de las preferencias de los receptores de cuidados profesionales de larga duración sobre el sexo de la persona cuidadora, presentados en este artículo, muestra una cierta persistencia de las construcciones sociales de género, sexualidad y cuidado en las mismas, ya que se continúan asociando al cuidado cualidades naturalizadas como femeninas (prácticas, detallistas, comunicativas) en especial para la higiene personal y la limpieza. Se justifica además la presencia de hombres en el cuidado con referencias a su fuerza física para la realización de las tareas que requieren esfuerzo y a la afinidad, cuando cuidan a hombres, en cuanto los temas de conversación. Por otra parte, las personas cuidadas, tanto hombres como mujeres, prefieren mayoritariamente para la realización de las tareas del cuidado relacionadas con la higiene personal, ser atendidas por personas del mismo sexo, por una cuestión de pudor. Aunque para el resto de tareas del cuidado, basándose en la formación de los cuidadores y en sus experiencias positivas de haber sido cuidadas por un hombre, manifiesten que les es indiferente el sexo de la persona cuidadora. Nuestro análisis de las preferencias de las personas cuidadas también muestra que el sexo de la persona cuidadora pasa a un segundo plano, tomando relevancia sus características personales, el trato y la profesionalidad. Nuestros resultados muestran también como en los contextos institucionales, se visibilizan de forma diferenciada la dimensión instrumental y la dimensión afectiva y emocional del cuidado. Poniendo en evidencia su complejidad y carácter relacional, así como el reto que supone que la profesionalización del cuidado, no vaya en detrimento de una atención individualizada y personalizada que tenga en cuenta las preferencias de las personas receptoras y que permita que el cuidado no sea vivido como una amenaza a la intimidad.

La profundización en el papel que las experiencias personales de cuidados realizados por 
hombres puedan tener en la reproducción o en el cambio de los estereotipos de género, requiere la realización de análisis comparativos de las preferencias de personas cuidadas en contextos institucionales y las de las personas cuidadas en su domicilio, tanto por personas remuneradas como por familiares. Una profundización que se podría realizar en futuras investigaciones sobre esta temática.

A pesar de las limitaciones de esta investigación, dado el tamaño y homogeneidad de la muestra, consideramos estos resultados pueden contribuir a mostrar la importancia de las opiniones de las personas receptoras de cuidados y el respeto a su intimidad, para la atención de sus necesidades de forma humanizada e individualizada, también en el cuidado profesional e institucionalizado. Nuestro trabajo puede también contribuir a mostrar la necesidad de la incorporación de la perspectiva de género al Trabajo Social, en concreto al análisis y a las intervenciones en situaciones de cuidado de larga duración, dada la importancia de los cuidados en la igualdad de género y la necesidad del compromiso y la implicación de los hombres en los trabajos de cuidados para afrontar la actual situación de crisis de los cuidados.

\section{Referencias bibliográficas}

Abellán, A., Esparza, C. y Pérez, J. (2011). Evolución y estructura de la población en situación de dependencia. Cuadernos de Relaciones Laborales, 29(1), 43-67.

Anxo, D. y Fagan, C. (2005). The family, the state, and now the market: the organisation of employment and working time in home care services for the elderly. Working in the Service Sector-a tale from different worlds. Londres: Routledge.

Báñez, T. (2017) "En el cuidado, a nosotros nos toca el papel de objetos". Discursos de las personas cuidadas sobre su capacidad de agencia de los cuidados y sus preferencias y relación con la persona cuidadora. En C. Guinot y A. Ferran (eds.), Trabajo social: arte para generar vínculos, Libro de Actas del III Congreso Internacional de trabajo social trabajo social: arte para generar vínculos, Universidad de Deusto, Bilbao: Publicaciones Universidad de Deusto.

Benería, L. (2011). Crisis de los cuidados, migración internacional y políticas públicas. En: C. Carrasco, C. Borderías y T. Torns, (eds.), El trabajo de cuidados. Historia, teoría y políticas. (pp. 359-389). Madrid: La Catarata.

Bodoque, Y., Roca, M. y Comas-d'Argemir, D. (2016). Hombres en trabajos remunerados de cuidados: género, identidad laboral y cultura del trabajo. Revista Andaluza de Antropología, 11, 67-91.

Caballero, A. (2010). La presencia del trabajo social en el Programa Individual de Atención (PIA) catalán. Revista de Servicios Sociales y Politica Social, 91, 107-117.

Cameron, C. y Moss, P. (2007). La atención a personas dependientes en Europa: conceptos actuales y perspectivas futuras. Psychosocial Intervention, 16(1), 7-22.

Canimas, J. (2008). De qué hablamos cuando hablamos de autonomía. Revista de Servicios Sociales y Politica Social, 84, 159-170.

Carrasquer Oto, P. (2013). El redescubrimiento de cuidados: algunas reflexiones desde la sociología. Cuadernos de Relaciones Laborales, 31(1), 91-113.

Colom, D. (2006). La dependencia: un reto de siempre convertido en un cúmulo de olvidos, despropósitos e intereses. Revista de Servicios Sociales y Politica Social, 73, 69-91.

Comas d'Argemir, D. (2016). Hombres cuidadores: Barreras de género y modelos emergentes. Psicoperspectivas, 15(3), 10-22.

Daly, M. y Lewis, J. (2000). The concept of social care and the analysis of contemporary welfare states. The British Journal of Sociology, 51(2), 281-298.

Erice, M. J. y Arraztoa, A. (2010). La escala de valoración social de la dependencia como instrumento para la coordinación sociosanitaria. Revista de Servicios Sociales y Politica Social, 91, 93-105.

Fuentes, M. C., y Moro, L. (2014). Trabajo Social y cuidadores informales: análisis de la situación actual y propuesta de intervención. Trabajo Social Hoy, 71, 43-62. doi:10.12960/TSH.2014.0002.

Himmelweit, S. (1995). The discovery of "unpaid work": the social consequences of the expansion of "work". Feminist Economics, 1(2), 1-19. 
Julve, M. (2006). Los cuidados invisibles de la dependencia. Revista de servicios sociales y politica social, $73,117-131$.

Lethbridge, J. (2011). Care Services for Older People in Europe - Challenges for Labour. Recuperado de: http://www.psiru.org/reports/care-services-older-people-europe-\%E2\%80\%93-challenges-labour [consultado el 5 de septiembre de 2016].

Ley 39/2006, de 14 de diciembre, de Promoción de la Autonomía Personal y Atención a las personas en situación de dependencia.

Mandell, B.R. (2010). The Crisis of Caregiving. Social Welfare Policy in the United States. Nueva York: Palgrave Macmillan.

Martínez, T., Díaz, B. y Sánchez, C. (2016). Respetando la intimidad: protección y trato cálido. Recomendaciones para la buena praxis en servicios sociales. Serie Ética y Servicios Sociales, ${ }^{\circ}$ 2. Oviedo: Consejería de Servicios y Derechos Sociales del Principado de Asturias. Recuperado de: https://www. asturias.es/Asturias/descargas/PDF_TEMAS/Asuntos\%20Sociales/Calidad/2016_11_22_guia_intimidad_proteccion_y_trato_calido.pdf [consultade el 5 de septiembre de 2016].

Martín-Serrano, E. (2009). La necesidad de un nuevo modelo de financiación del Sistema de Dependencia: análisis de los desequilibrios actuales que sobrefinancia las prestaciones económicas respecto de los servicios y no incentiva la plena implantación de las coberturas. Revista de Servicios Sociales y Politica Social, 88, 105-121

Organización Mundial de la Salud. (OMS) (2000) Hacia un consenso internacional sobre los programas de cuidados de larga duración para las personas mayores. Recuperado de: http://apps.who.int/iris/handle/10665/66542

Oria, M.R. (2009). Reflexión sobre sistemas de evaluación de la situación de dependencia desde la práctica profesional del trabajo social. Instrumento para la aplicación del Servicio de Ayuda a Domicilio. Servicios Sociales y Política Social, 88, 55-63

Pérez-Orozco, A. (2006). Amenaza tormenta: la crisis de los cuidados y la reorganización del sistema económico, Revista de Economía Crítica, 5, 7-37.

Pi Giménez, V. (2012). El trabajo social se viste de naranja. Servicios sociales y politica social, 99, 109-120.

Prieto, C. y Serrano, A. (2013). Los cuidados entre el trabajo y la vida. Cuadernos de Relaciones Laborales, 31(1), 11-17.

Recio, C., Moreno, S., Borràs, V. y Torns, T. (2015). La profesionalización de sector de los cuidados. Zerbitzuan, 60, 179-193.

Rimbau, C. (2014). La gestión de caso. Conceptualización, contexto y presencia social actual. Servicios sociales y política social, 105, 33-44.

Roca, M. y Bodoque, Y. (2017). Trabajadores del cuidado: estrategias de contratación de hombres en trabajos remunerados de cuidados. En: G. Jaraíz (comp.), Actas VI Congreso de la Red Española de Políticas sociales, 16-17 de febrero, Sevilla (España) (En prensa).

Rodríguez, C. (2013). Las familias y los cuidados a las personas mayores dependientes: entre la reciprocidad y la ambivalencia. Cuadernos de Trabajo Social, 26(2), 349-358.

Romero, R. (2012). Los Servicios Sociales municipales ante el Sistema de Autonomía y Atención a la Dependencia. Aportaciones desde la perspectiva y proximidad del Trabajo Social. Servicios sociales y política social, 98, 101-112.

Soronellas, M. y Comas-D'Argemir, D. (2017). Hombres cuidadores de personas adultas dependientes. ¿Estrategias ante la crisis o nuevos agentes en los trabajos de cuidados? En: G. Jaraíz, G. (comp.), Actas VI Congreso de la Red Española de Políticas sociales, 16-17 de febrero, Sevilla (España) (En prensa).

Tobío, C., Agulló, M. S., Gómez, M.V. y Martín, M.T. (2010). El cuidado de las personas. Un reto para el siglo XXI. Barcelona: Fundación La Caixa. Recuperado de: https://obrasocial.lacaixa.es/deployedfiles/ obrasocial/Estaticos/pdf/Estudios_sociales/vol28_completo_es.pdf [consultado el 25 de septiembre de 2016].

Torns, T., Borràs, V., Moreno, S. y Recio, C. (2014). Nuevas profesiones para la organización social del cuidado cotidiano. Madrid: Instituto de la Mujer, NIPO, 685-14. Recuperado de: http://www.inmujer. gob.es/areasTematicas/estudios/estudioslinea2014/docs/Nuevas_profesiones_organizacion_social.pdf [consultado el 25 de septiembre de 2016].

Wellin, C. y Jaffe, D. (2004). In search of "personal care": Challenges to identity support in residential care for elders with cognitive illness. Journal of Aging Studies, 18(3), 275-295. 
Williams, F. (2012). Care relations and public policy: social justice claims and social investment frames.

Families, Relationships and Societies, 1 (1), 103-119

Zelizer, V. (2009). La negociación de la intimidad, Buenos Aires: Fondo de Cultura Económica. 